\title{
Microhabitat preference of frogs at Similajau National Park, Sarawak, Malaysia
}

\begin{abstract}
Tropical heath forest occurs widely in many places of Borneo. Similajau National Park is one of the locations with heath forest. Because of insufficient data on the anurans of heath forest in Similajau National Park, a survey was carried out in the beginning of August 2014 until January 2015. The objectives of the study were to compare the anuran species diversity between two different study plots and to determine anuran species microhabitat preferences. The sampling method involved a 300m transects line and 240 quadrats method (5m x 5m) at both study sites. A total of 23 species of frogs (299 individuals) belonging to six frog families were detected. The study site was dominated by anurans of the family Dicroglossidae $(48.8 \%)$ followed by Rhacophoridae (24.7\%), Ranidae (17.7\%), Ceratobatrachidae (4.7\%), Microhylidae (1.3\%) and Bufonidae (2.7\%). Four endemic Borneo frogs, namely Ingerophyrus divergens, Limnonectes ingeri, Kalophrynus intermedius, and Philautus tectus were among anurans captured in this study. The Shannon Wiener Diversity Index, H' at forest site was higher than the non-forest site $(2.48 \pm 0.06$ vs. $1.77 \pm 0.05)$. There was no dominant species at forest sites, which was reflected by the low Simpson Index, D, $0.091( \pm 0.01)$. Conversely, the same index at the non-forest site was higher with $0.201( \pm 0.04)$. This is apparent with the presence of Fejervarya cancrivora that made up about $20 \%$ of the total frogs sampled in the area. Habitat preferences associated with the recovered species from this are discussed in this paper.
\end{abstract}

Keyword: Diversity; Frog occurrence; Kerangas forest; Non-forest; Similajau National Park 